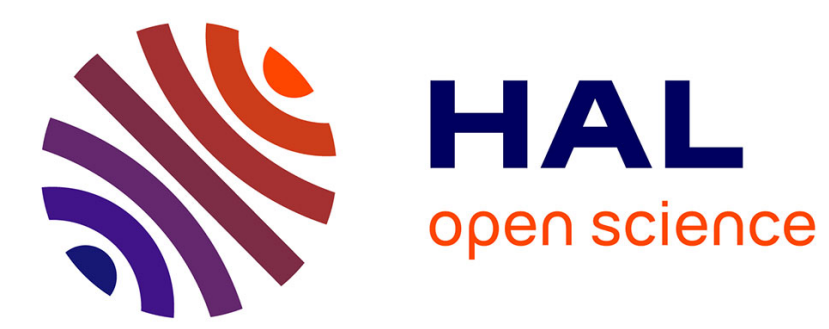

\title{
Giant spontaneous resistivity jumps in La0.825Ce0.175Fe12B6
}

L. V. B. Diop, O. Isnard

\section{To cite this version:}

L. V. B. Diop, O. Isnard. Giant spontaneous resistivity jumps in La0.825Ce0.175Fe12B6. Applied Physics Letters, 2021, 119 (3), pp.032403. 10.1063/5.0059731 . hal-03325794

\section{HAL Id: hal-03325794 \\ https://hal.science/hal-03325794}

Submitted on 25 Aug 2021

HAL is a multi-disciplinary open access archive for the deposit and dissemination of scientific research documents, whether they are published or not. The documents may come from teaching and research institutions in France or abroad, or from public or private research centers.
L'archive ouverte pluridisciplinaire HAL, est destinée au dépôt et à la diffusion de documents scientifiques de niveau recherche, publiés ou non, émanant des établissements d'enseignement et de recherche français ou étrangers, des laboratoires publics ou privés. 


\title{
Giant spontaneous resistivity jumps in $\mathrm{La}_{0.825} \mathrm{Ce}_{0.175} \mathrm{Fe}_{12} \mathrm{~B}_{6}$
}

\author{
L.V.B. Diop ${ }^{1 \dagger}$ and O. Isnard ${ }^{2}$ \\ ${ }^{1}$ Université de Lorraine, CNRS, IJL, F-54000 Nancy, France \\ ${ }^{2}$ Université Grenoble Alpes, CNRS, Institut NEEL, 25 rue des martyrs, BP166X, F-38042 Grenoble, \\ France
}

We report the observation of spontaneous and very sharp resistivity jumps at low temperatures in the antiferromagnetic itinerant-electron system $\mathrm{La}_{0.825} \mathrm{Ce}_{0.175} \mathrm{Fe}_{12} \mathrm{~B}_{6}$. This intermetallic compound undergoes multiple magnetic transformations, antiferromagneticferromagnetic (AFM-FM) and ferromagnetic-paramagnetic (FM-PM), triggered by changes in both temperature and applied magnetic field. The magnetoresistance isotherms display irreversible abrupt steps at $T \leq 4 \mathrm{~K}$, whereas the field dependence of the resistivity becomes smooth above $4 \mathrm{~K}$. Meanwhile, the evolution with time of the electrical resistivity exhibits a huge spontaneous jump after an incubation time when both the applied magnetic field and temperature are constant. A giant negative magnetoresistance $(\mathrm{MR}=-71 \%)$ is discovered associated with the magnetic-field-induced first-order AFM-FM transition.

Itinerant electron metamagnetism (IEM) is an essential physical phenomenon, depicting magnetic behaviors of some $3 \mathrm{~d}-4 \mathrm{f}$ intermetallic compounds, manifesting potentially important functionalities such as colossal magnetoresistance, large magnetostriction and giant magnetocaloric effects ${ }^{1-4}$. One interesting example of IEM is $\mathrm{LaFe}_{12} \mathrm{~B}_{6}$ intermetallic which occupies a special place among rare-earth iron-rich compounds. $\mathrm{LaFe}_{12} \mathrm{~B}_{6}$ presents exotic magnetic behavior, many anomalous features and intriguing physical properties. Unconventional multistep metamagnetic transitions were recently discovered in the ternary system $\mathrm{LaFe}_{12} \mathrm{~B}_{6}{ }^{5-8}$. These exceptional metamagnetic phase transitions are characterized by ultrasharp steps followed by plateaus leading to an avalanche- like (or staircase-like) magnetization process. Neutron diffraction studies revealed an unusual amplitude-modulated spin configuration defined by a magnetic propagation vector $\mathbf{k}=(1 / 4,1 / 4,1 / 4)$ and remarkably weak Fe moment $(0.43 \mu \mathrm{B})$ in the antiferromagnetic ground state ${ }^{5}$. Moreover, $\mathrm{LaFe}_{12} \mathrm{~B} 6$ exhibits extraordinary low Néel temperature $T_{\mathrm{N}}=36 \mathrm{~K}$ for an Fe-rich alloy, a critical point in the magnetic phase diagram ${ }^{5}$, both normal and inverse magnetocaloric effects ${ }^{9}$, and large magnetovolume effects ${ }^{10}$. These singular properties not only tendered the development of

${ }^{\dagger}$ leopold.diop@univ-lorraine.fr 
theoretical models and experiments under extreme conditions ${ }^{10-14}$, but also highlighted the potential interest of $\mathrm{LaFe}_{12} \mathrm{~B}_{6}$ material in future low-temperature energy technologies. $\mathrm{LaFe}_{12} \mathrm{~B}_{6}$ constitutes a phenomenal playground for materials physics because of the extreme sensitivity of its physical properties to moderate hydrostatic pressure ${ }^{10}$ and chemical substitution ${ }^{8}$ that produces the effect of "chemical pressure". Among the ternary system $R T_{12} \mathrm{~B}_{6}$ (where $R$ stands for a rare-earth atom and $T$ is a $3 d$ transition metal element $\mathrm{Co}$ or $\mathrm{Fe}$ ), $\mathrm{LaFe}_{12} \mathrm{~B}_{6}$ is the sole stable Fe-based phase of the 1:12:6 family ${ }^{13-15}$. By contrast, the $R \mathrm{Co}_{12} \mathrm{~B}_{6}$ alloys are stable along the entire lanthanide series. The intermetallic system $\mathrm{LaFe}_{12} \mathrm{~B}_{6}$ is unique among the $R T_{12} \mathrm{~B}_{6}$ family in being an antiferromagnet with a magnetic transition temperature much smaller than the Curie point of the Co-based $R \mathrm{Co}_{12} \mathrm{~B}_{6}$ ferro- $(R=\mathrm{Y}, \mathrm{La}-\mathrm{Sm})$ or ferri- $(R=\mathrm{Gd}-\mathrm{Tm})$ magnets $\left(T_{\mathrm{C}}=134\right.$ - $162 \mathrm{~K})^{15}$ and an order of magnitude smaller when compared to the magnetic ordering temperature of any rare-earth iron-rich binary intermetallic. Interestingly, extraordinary magnetotransport effects have been most recently observed in $R \mathrm{Co}_{12} \mathrm{~B}_{6}$ alloys with $R=\mathrm{Y}$, Gd and $\mathrm{Ho}^{16}$.

In our earlier investigation of the magnetic properties of the $\mathrm{La}_{1-x} \mathrm{Ce}_{x} \mathrm{Fe}_{12} \mathrm{~B}_{6}$ series of compounds, we have shown that the antiferromagnetic (AFM) ground state and the paramagnetic (PM) phase get transformed into a ferromagnetic (FM) state via a field-induced first-order metamagnetic phase transition ${ }^{8}$. In the present letter, we report on the occurrence of huge spontaneous resistivity jump across the magnetic transition in $\mathrm{La} 0.825 \mathrm{C}_{\mathrm{e} 0.175} \mathrm{Fe}_{12} \mathrm{~B}_{6}$ in conditions where both the applied magnetic field and temperature are kept constant.

The sample used in this study was taken from the same batch as that employed previously in magnetization experiments. Details on the synthesis and subsequent characterization of the $\mathrm{La} 0.825 \mathrm{C}_{\mathrm{e} 0.175} \mathrm{Fe}_{12} \mathrm{~B}_{6}$ intermetallic compound are described in Ref. 8. For the resistivity and magnetoresistance measurements, the specimen was cut in parallelepiped shape using diamond saw and then smooth and flat surfaces were prepared by polishing. The electrical contacts on the sample surface were made by fixing thin platinum wires using silver paste. The experiments were conducted using the conventional four-point contact method at a constant dc current of $10 \mathrm{~mA}$ over the temperature range between 2.5 and $150 \mathrm{~K}$ in a superconducting coil providing a maximum field of $8 \mathrm{~T}$. Measurements were performed according to the following configuration: magnetic field vector oriented perpendicular to the direction of electrical current $(\boldsymbol{H} \perp \boldsymbol{i})$. At each measurement point, the dc electrical current was applied in opposite polarities in order to get rid of possible thermals. Zero-field cooled (ZFC) and field cooled (FC) experimental procedures were employed for the isofield measurements.

Figure 1 displays the temperature dependence of the electrical resistivity, $\rho(T)$, of 
La0.825 $\mathrm{Ce}_{0.175} \mathrm{Fe}_{12} \mathrm{~B}_{6}$ in $\mathrm{ZFC}$ and $\mathrm{FC}$ modes under various magnetic fields between 0 and $8 \mathrm{~T}$. At high temperatures, the isofield $\rho(T)$ plots present a strongly linear variation which is indicative of the metallic character. In zero magnetic field $\left(\mu_{0} H=0 \mathrm{~T}\right)$, both heating and cooling resistivity functions are nearly identical; indicating that the direction of the temperature change does not affect the mechanisms responsible for charge-carrier scattering and their concentration. No perceptible anomaly is observed at the Néel temperature $T_{\mathrm{N}}$ associated with the second-order AFM-PM magnetic transition. The large change in resistivity around $86 \mathrm{~K}$ upon heating under a magnetic field of $\mu_{0} H=8 \mathrm{~T}$ arises from magnetic phase transformation between the FM (low resistivity) and PM (high resistivity) phases. This value of the magnetic ordering temperature is in excellent agreement with the Curie temperature $T_{\mathrm{C}}$ derived from the thermal dependence of the magnetization reported in Ref. 8. The $6 \mathrm{~T}$ temperature-dependent electrical resistivity curve show the same behavior as that obtained at $8 \mathrm{~T}$ except for a negative shift of $T_{\mathrm{C}}$ toward lower temperatures. In the vicinity of $T_{\mathrm{C}}$, the increase of external field diminishes the electrical resistivity because the spin scattering is reduced by the magnetic-field-induced alignment of the local magnetic moments. The tremendous resistivity variation at $T_{\mathrm{C}}$ reflects a strong interaction of Fe magnetic moments with conduction electrons.

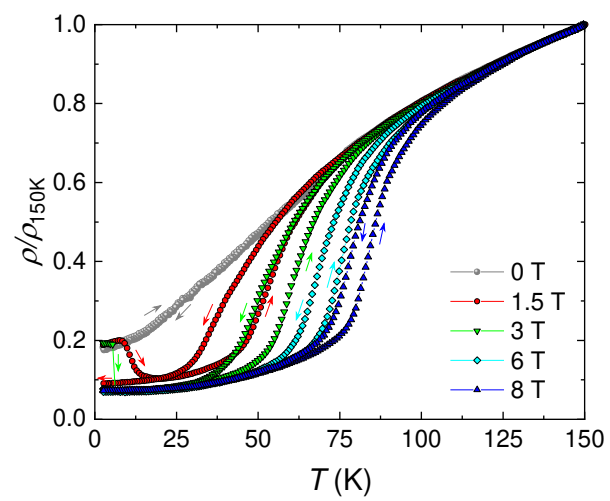

FIG. 1. Temperature dependence of the relative electrical resistivity of $\mathrm{La}_{0.825} \mathrm{Ce}_{0.175} \mathrm{Fe}_{12} \mathrm{~B}_{6}$ on heating and cooling in various applied magnetic fields. Both ZFC and FC data are marked by the same symbols. The arrows indicate the direction of the temperature change.

However, the temperature dependence of the resistivity measured in $1.5 \mathrm{~T}$ differs considerably from the behavior observed in zero and high magnetic fields. The $1.5 \mathrm{~T} \rho(T)$ plot exhibits a strong splitting between ZFC and FC measuring protocols and shows an even more interesting thermal evolution. Upon increasing temperature from the initial AFM ground state 
at $2.5 \mathrm{~K}$, the resistivity first decreases at the onset of the FM order followed by a plateau and before increasing again at high temperature; generating a basin-shaped behavior. This peculiar thermal variation of the resistivity is associated with the existence of both low-temperature AFM-FM and high-temperature FM-PM phase transformations. Another noteworthy experimental observation in the resistivity curves of Fig. 1 is the remarkably large temperature hysteresis near the FM-PM transformation, $\approx 13 \mathrm{~K}$ for $\mu_{0} H=1.5 \mathrm{~T}$, which emphasizes the firstorder nature of the magnetic phase transition. Unexpectedly, the 3 T ZFC $\rho(T)$ curve presents an ultrasharp step at which the normalized resistivity drops abruptly from 0.19 to 0.07 when temperature is increased by only $0.7 \mathrm{~K}$. This sudden resistivity jump is strikingly similar to that seen in the $3 \mathrm{~T}$ thermomagnetic curve of the thermally demagnetized compound and demonstrates the strong correlation between electronic transport and magnetism in $\mathrm{La}_{0.825} \mathrm{Ce}_{0.175} \mathrm{Fe}_{12} \mathrm{~B}_{6}$. The pronounced resistivity change at the order-order $\mathrm{AFM} \rightarrow \mathrm{FM}$ phase transition can be ascribed to the difference in the strength of the scattering of the conduction electrons by the AFM and FM magnons and by the phonons. Our results undoubtedly proves that the scattering in the FM spin structure is smaller than that in the AFM magnetic arrangement.

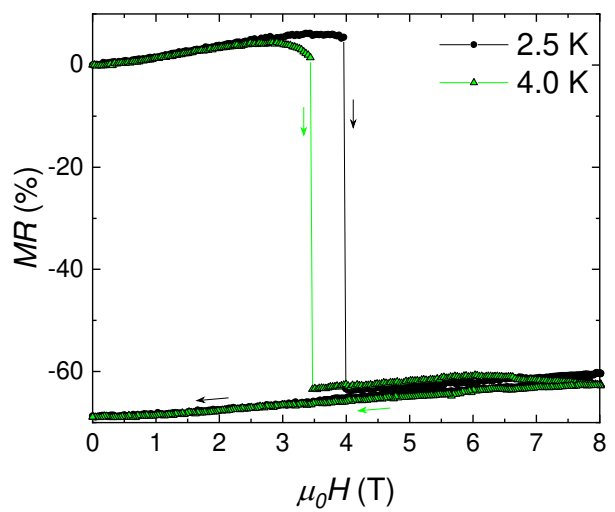

FIG. 2. Magnetoresistance isotherms of $\mathrm{La}_{0.825} \mathrm{Ce}_{0.175} \mathrm{Fe}_{12} \mathrm{~B}_{6}$ at 2.5 and $4 \mathrm{~K}$.

To further elucidate the interplay between charge and magnetic degrees of freedom in this intermetallic system, the field dependence of the electrical resistivity was recorded at several fixed temperatures. From these isothermal measurements, the magnetoresistance ratio MR was assessed as $\left[\rho\left(\mu_{0} H, T\right)-\rho(0, T)\right] / \rho(0, T)$. Magnetoresistance isotherms of the zero- 
magnetic-field cooled compound are illustrated in Figs. 2-3 for different representative temperature ranges. As follows from Fig. 2, during the first application of the magnetic field at very low temperatures, the resistivity initially increases and then displays a steep discontinuity at a threshold featured by a critical field. The sharp stepwise resistivity change occurs in the same magnetic field interval where a large discrete jump was detected in the virgin magnetization curves. This abrupt and dramatic change in the magnetoresistance is due to the magnetic-field-induced first-order AFM-FM transition, with the forced FM phase having a smaller electrical resistivity when compared with that of the AFM ground state. The subsequent decreasing-field branch shows no anomalies since $\mathrm{La} 0.825 \mathrm{Ce} 0.175 \mathrm{Fe}_{12} \mathrm{~B}_{6}$ remains in the fielddriven FM state as proved by the magnetization data $^{8}$. The initial resistivity value is not recovered after removal of the applied magnetic field; leading to the presence of a remanent (nonzero) magnetoresistance. This feature attests to the complete irreversibility of the AFMFM phase transformation in the very low temperature regime. In order to restore the original AFM ground state (virgin state), and therefore, the initial value of the electrical resistivity, the intermetallic compound $\mathrm{La} 0.825 \mathrm{Ce}_{0.175} \mathrm{Fe}_{12} \mathrm{~B}_{6}$ should be warmed up beyond $T_{\mathrm{C}}$ and then cooled down in zero applied magnetic field.

At temperatures exceeding $5 \mathrm{~K}$, the isothermal magnetoresistance curves present gradual changes across both AFM-FM and PM-FM transformations differently from the discontinuous behavior seen below $5 \mathrm{~K}$. The magnetic transitions are accompanied by a huge field hysteresis which is one of the signatures of a first-order transformation. In addition, the hysteretic character and the irreversibility/reversibility depend strongly on the temperature range. We exemplify in Fig. 3 the isothermal magnetoresistance plots in two distinct representative temperature intervals: between 5 and $30 \mathrm{~K}$ (lower panel) and $T \geq 40 \mathrm{~K}$ (upper panel). In the AFM ground state, for instance at $20 \mathrm{~K}$, the magnetoresistance ratio drastically decreases as the system endures a magnetic transition to the FM state yielding a giant negative MR effect. The magnetoresistance variation due to the field-induced AFM-FM metamagnetic phase transition amounts to $\mathrm{MR}=-71 \%$ at $20 \mathrm{~K}$. No transition is detected in the reverse leg and MR keeps a nearly constant value down to the zero field point; clearly indicating the irreversible nature of the magnetic transition below $30 \mathrm{~K}$. In the temperature range between 40 and $70 \mathrm{~K}$, the downward-field curve diverts from the pure FM behavior and a metamagnetic-like transition takes place at lower magnetic field. In this temperature region, the PM-FM phase transition is partially reversible; a fraction of the compound recovers the PM state when the applied magnetic field is brought back to zero. That is, both irreversible and reversible magnetic phase transformations exist in this temperature interval and the proportion of $\mathrm{La} 0.825 \mathrm{Ce}_{0.175} \mathrm{Fe}_{12} \mathrm{~B}_{6}$, 
which undergoes the irreversible transformation, declines with increasing temperature. The transition field of the downward-field path, $\mu_{0} H_{\text {crd }}$, varies monotonically with temperature. In contrast, the thermal evolution of the critical field obtained for the ascending-field scan, $\mu_{0} H_{\text {cra, }}$, is nonmonotonic. Below $20 \mathrm{~K} \mu_{0} H_{\text {cra }}$ increases as the temperature is lowered whereas it exhibits the inverse behavior at higher temperatures. Below $20 \mathrm{~K}$, the AFM-FM critical magnetic field rises upon cooling due to the enhancement of the negative exchange interactions and the reduction of the thermal fluctuations of the spins and elasticity of the crystal lattice in the AFM ground state $\mathrm{e}^{5,8,17}$. This results in the increase of both the critical magnetic transition field required to complete the metamagnetic transformation from one phase to another, and the free energy difference between the AFM and FM states.

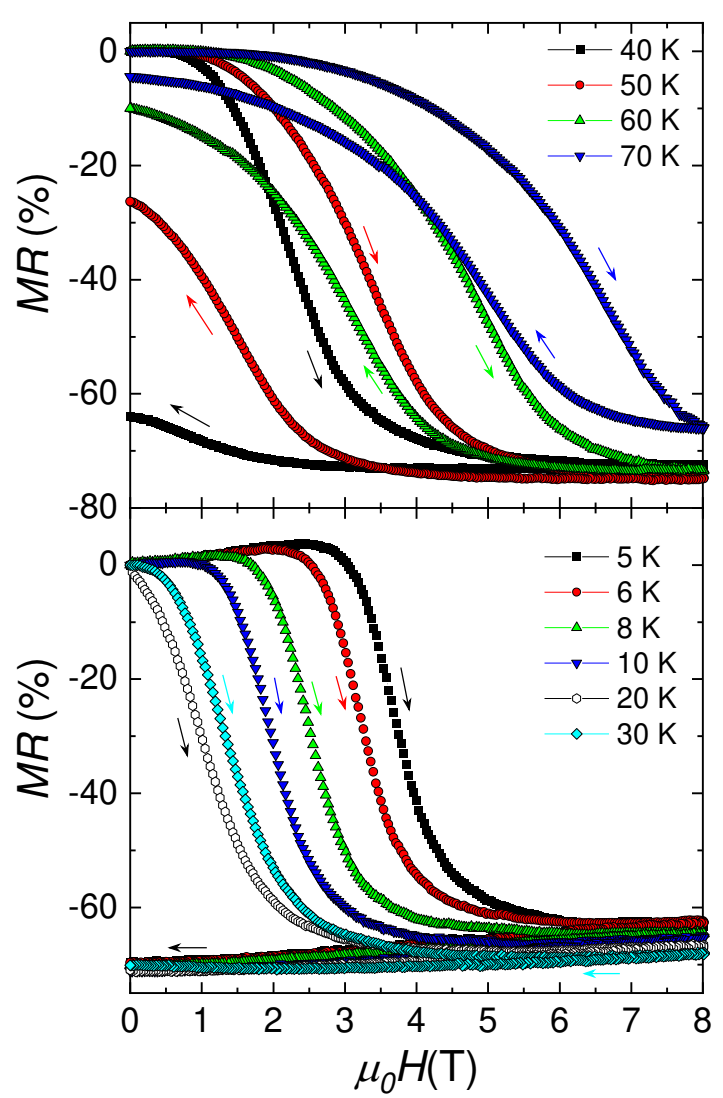

FIG. 3. Magnetoresistance isotherms of $\mathrm{La}_{0.825} \mathrm{Ce}_{0.175} \mathrm{Fe}_{12} \mathrm{~B}_{6}$ for the temperature intervals 5 to $30 \mathrm{~K}$ (lower panel) and 40 to $70 \mathrm{~K}$ (upper panel). 


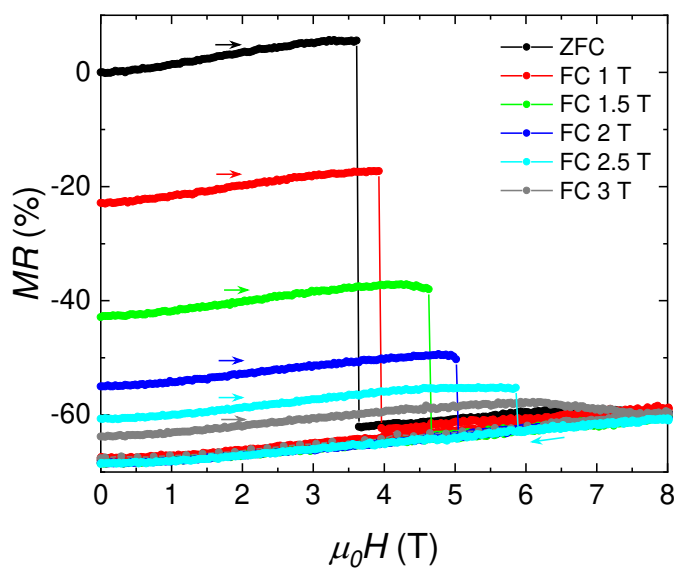

FIG. 4. Magnetoresistance isotherms of $\mathrm{La}_{0.825} \mathrm{Ce}_{0.175} \mathrm{Fe}_{12} \mathrm{~B}_{6}$ recorded at $2.5 \mathrm{~K}$ after cooling the sample in different magnetic fields.

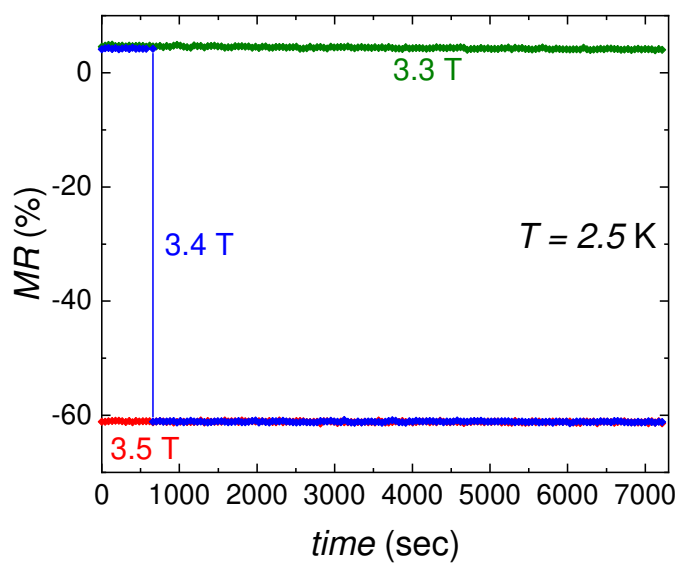

FIG. 5. Time dependence of the magnetoresistance collected at the indicated applied fields for $\mathrm{La}_{0.825} \mathrm{Ce}_{0.175} \mathrm{Fe}_{12} \mathrm{~B}_{6}$ at $2.5 \mathrm{~K}$.

As demonstrated in our previous magnetization $\operatorname{study}^{8}$, field cooling alters significantly the relative concentration of the different magnetic phases - AFM, FM, and PM. By analogy to the magnetic data, we investigated the influence of the field strength applied during the cooling process on the electrical resistivity. For such investigations, the alloy was cooled in the presence of a positive applied magnetic field $\left(\mu_{0} H>0\right)$ from high temperatures (paramagnetic region) down to $2.5 \mathrm{~K}$. After stabilizing the measurement temperature of $2.5 \mathrm{~K}$, the cooling field was reduced to zero, and subsequently the resistivity was recorded as a function of magnetic field 
(Fig. 4). This magnetic field cooling process reduces the low-field resistivity owing to the growth of the FM phase concentration to the detriment of the AFM component. The critical field at which the step transition occurs is fully mastered by the percentage of the FM phase in the compound. The resistivity jump is shifted toward higher magnetic fields upon increasing the magnitude of the magnetic field applied during the cooling procedure.

In view of the metastability of the different magnetic phases, time-dependent phenomena (relaxation effects) were examined to probe more directly the dynamics of the resistivity jump. The relaxation effects were investigated at $2.5 \mathrm{~K}$ and in applied magnetic fields slightly below and above the critical field associated with the step-like transition. Prior to these time dependence experiments, the compound was cooled from room temperature to $2.5 \mathrm{~K}$ without an applied magnetic field. Once the temperature of $2.5 \mathrm{~K}$ is stabilized, a magnetic field is applied afterward the electrical resistivity is measured versus time (duration of $7200 \mathrm{~s}$ ). The same protocol was repeated several times by changing the field strength in steps of $0.1 \mathrm{~T}$. The corresponding results are reported in Fig. 5. For a magnetic field value of 3.4 T, MR decreases spontaneously from $\approx 4 \%$ to $\approx-61 \%$, a change that we assign to the sudden formation of $\mathrm{FM}$ domains inside the AFM matrix. The 3.4 T curve exhibits giant resistive relaxation effects. Note that this spectacular and abrupt resistivity change during isothermal holding takes place over a time period smaller than the time interval separating two consecutive experimental points, i.e. $<40 \mathrm{~s}$. The most intriguing feature in the present data is the huge impulsive step in electrical resistivity after an incubation time of $660 \mathrm{~s}$ when both the applied magnetic field and temperature are kept constant. The quality of the temperature stabilization was examined during the isothermal holding $(2.500 \pm 0.008 \mathrm{~K})$. Additionally, the magnetic field is equally expected to be very stable over the entire duration of the resistive relaxation experiments since it is applied by a superconducting coil in a persistent mode. These exceptional relaxation phenomena observed at $3.4 \mathrm{~T}$ in $\mathrm{La} 0.825 \mathrm{Ce}_{0.175} \mathrm{Fe}_{12} \mathrm{~B}_{6}$ are reminiscent of an explosive instability, where the resistivity of the intermetallic compound endures a colossal change in a very short time interval. They also show a close analogy to the peculiar time evolution of the electrical resistivity in standard martensitic transitions where an abrupt jump is detected after a holding time of about $1020 \mathrm{~s}$ for the Fe-31.7at.\%Ni alloy ${ }^{18}$.

Even though the giant spontaneous jump is seen on both magnetic and resistive relaxation data for $\mathrm{La}_{0.825} \mathrm{Ce}_{0.175} \mathrm{Fe}_{12} \mathrm{~B}_{6}$ compound, yet, there is a large discrepancy in the transition time. The incubation time considerably differs from $660 \mathrm{~s}$ to $4860 \mathrm{~s}$ for resistive and magnetic ${ }^{8}$ isothermal holding, respectively. This clearly indicates that the characteristic time associated with the sudden step is not a material constant. 
To summarize, an unusual and intriguing stepwise resistive relaxation is found in the itinerant-electron metamagnetic compound $\mathrm{La}_{0.825} \mathrm{Ce}_{0.175} \mathrm{Fe}_{12} \mathrm{~B}_{6}$. At constant temperature and applied magnetic field, a giant spontaneous resistivity jump separating two plateaus takes place after an incubation period. This unique step-like feature endorses the existence of a similarity between the metamagnetic transformation in $\mathrm{La} 0.825 \mathrm{Ce}_{0.175} \mathrm{Fe}_{12} \mathrm{~B}_{6}$ and the isothermal martensitic transition in some metallic alloys. A remarkably large negative magnetoresistance $\mathrm{MR}=-71 \%$ is observed. Our findings shed light on the phenomenon of avalanche-like metamagnetic transitions found in different systems.

\section{DATA AVAILABILITY}

The data that support the findings of this study are available from the corresponding author upon reasonable request.

\section{REFERENCES}

${ }^{1}$ D.P. Kozlenko, E. Burzo, P. Vlaic, S.E. Kichanov, A.V. Rutkauskas, and B.N. Savenko, Sci. Rep. 5, 8620 (2015).

${ }^{2}$ A. Fujita, S. Fujieda, Y. Hasegawa, and K. Fukamichi, Phys. Rev. B 67, 104416 (2003).

${ }^{3}$ H. Yamada, and T. Goto Phys. Rev. B 68, 184417 (2003).

${ }^{4}$ N.H. Duc, D.T. Kim Anh, and P.E. Brommer, Physica B 319, 1 (2002).

${ }^{5}$ L.V.B. Diop, O. Isnard, and J. Rodríguez-Carvajal, Phys. Rev. B 93, 014440 (2016).

${ }^{6}$ S. Fujieda, K. Fukamichi, and S. Suzuki, J. Magn. Magn. Mater. 421, 403 (2017).

${ }^{7}$ L.V.B. Diop, and O. Isnard, Appl. Phys. Lett. 108, 132401 (2016).

${ }^{8}$ L.V.B. Diop, and O. Isnard, Phys. Rev. B 97, 014436 (2018).

${ }^{9}$ L.V.B. Diop, and O. Isnard, J. Appl. Phys. 119, 213904 (2016).

${ }^{10}$ L.V.B. Diop, O. Isnard, Z. Arnold, J.P. Itié, J. Kastil, and J. Kamarad, Solid State Comm. 252, 29 (2017).

${ }^{11}$ G.I. Miletic, and Z. Blazina, J. Magn. Magn. Mater. 323, 2340 (2011).

${ }^{12}$ G.I. Miletic, and Z. Blazina, J. Alloys Compd. 430, 9 (2007).

${ }^{13}$ M. Rosenberg, T. Sinnemann, M. Mittag, and K.H.J. Buschow, J. Alloys Compd. 182, 145 (1992).

${ }^{14}$ Q.A. Li, C.H. de Groot, F.R. de Boer, and K.H.J. Buschow, J. Alloys Compd. 256, 82 (1997). ${ }^{15}$ M. Mittag, M. Rosenberg, and K.H.J. Buschow, J. Magn. Magn. Mater. 82, 109 (1989).

${ }^{16}$ F. Mesquita, S.G. Magalhaes, P. Pureur, L.V.B. Diop, and O. Isnard, Phys. Rev. B 101, 224414 (2020). 
${ }^{17}$ E.M. Levin, K.A. Gschneidner, Jr., and V.K. Pecharsky, Phys. Rev. B 65, 214427 (2002).

${ }^{18}$ T. Kakeshita, J. Katsuyama, T. Fukuda, and T. Saburi, Mater. Sci. Eng. A 312, 219 (2001). 


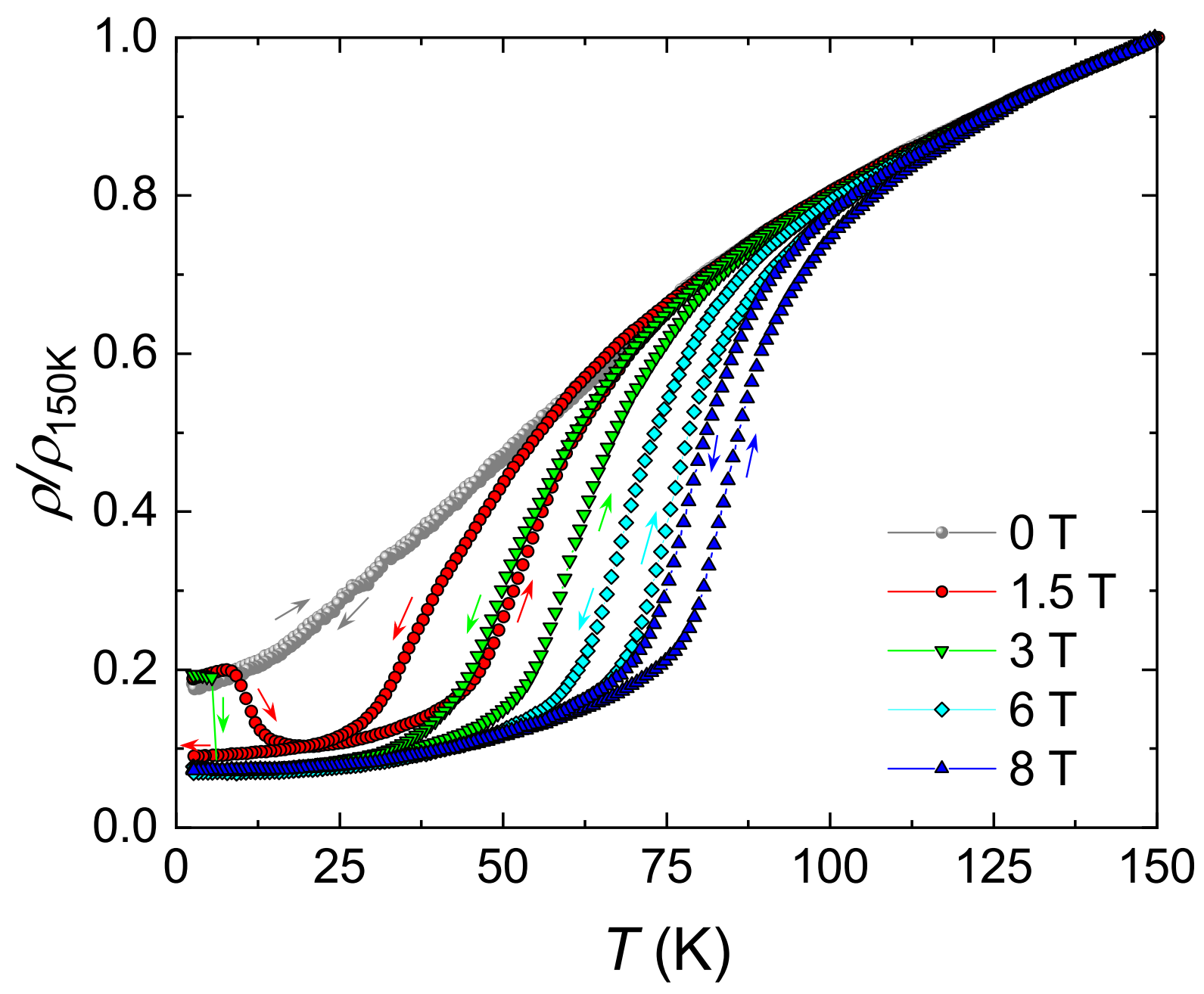

는 


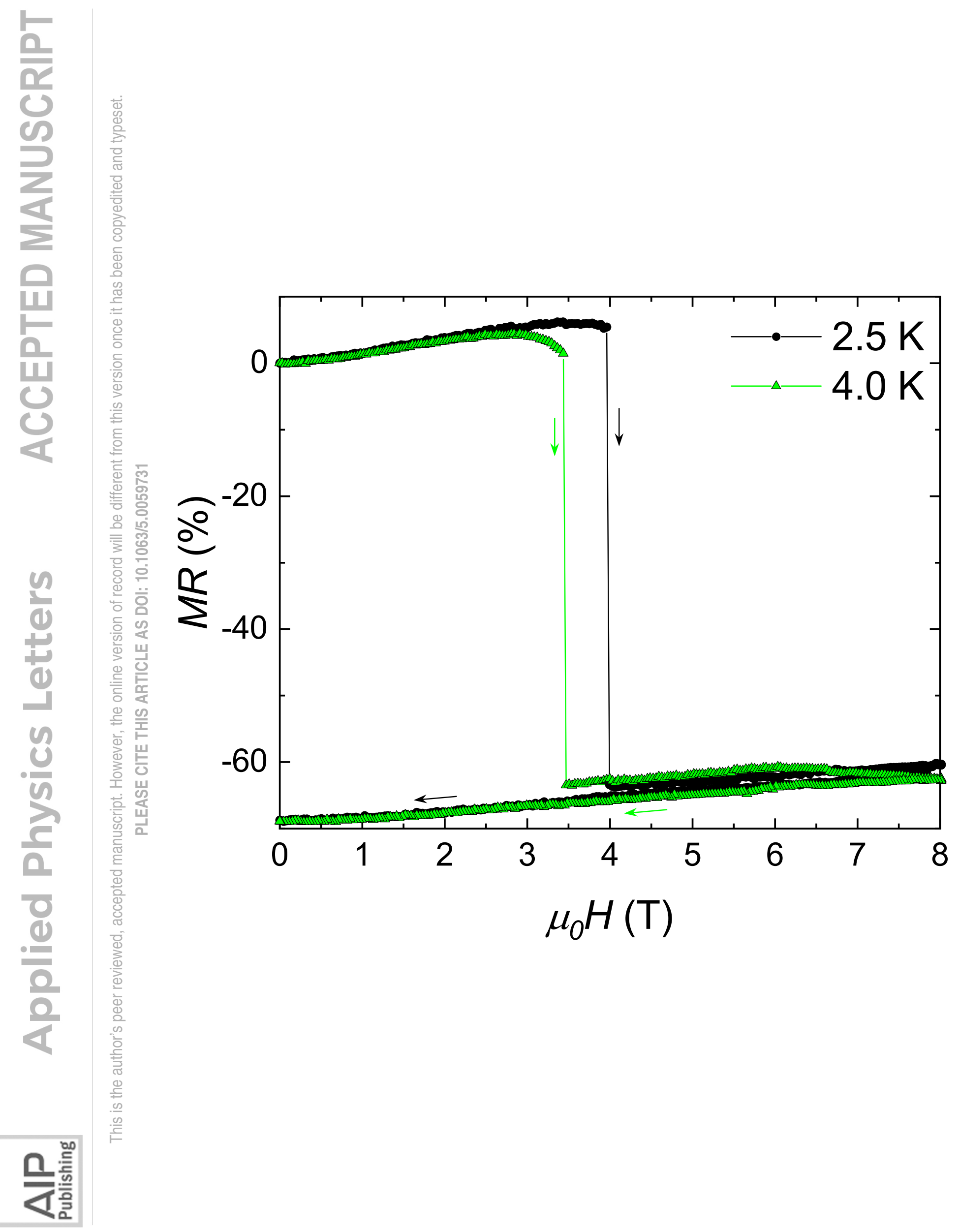



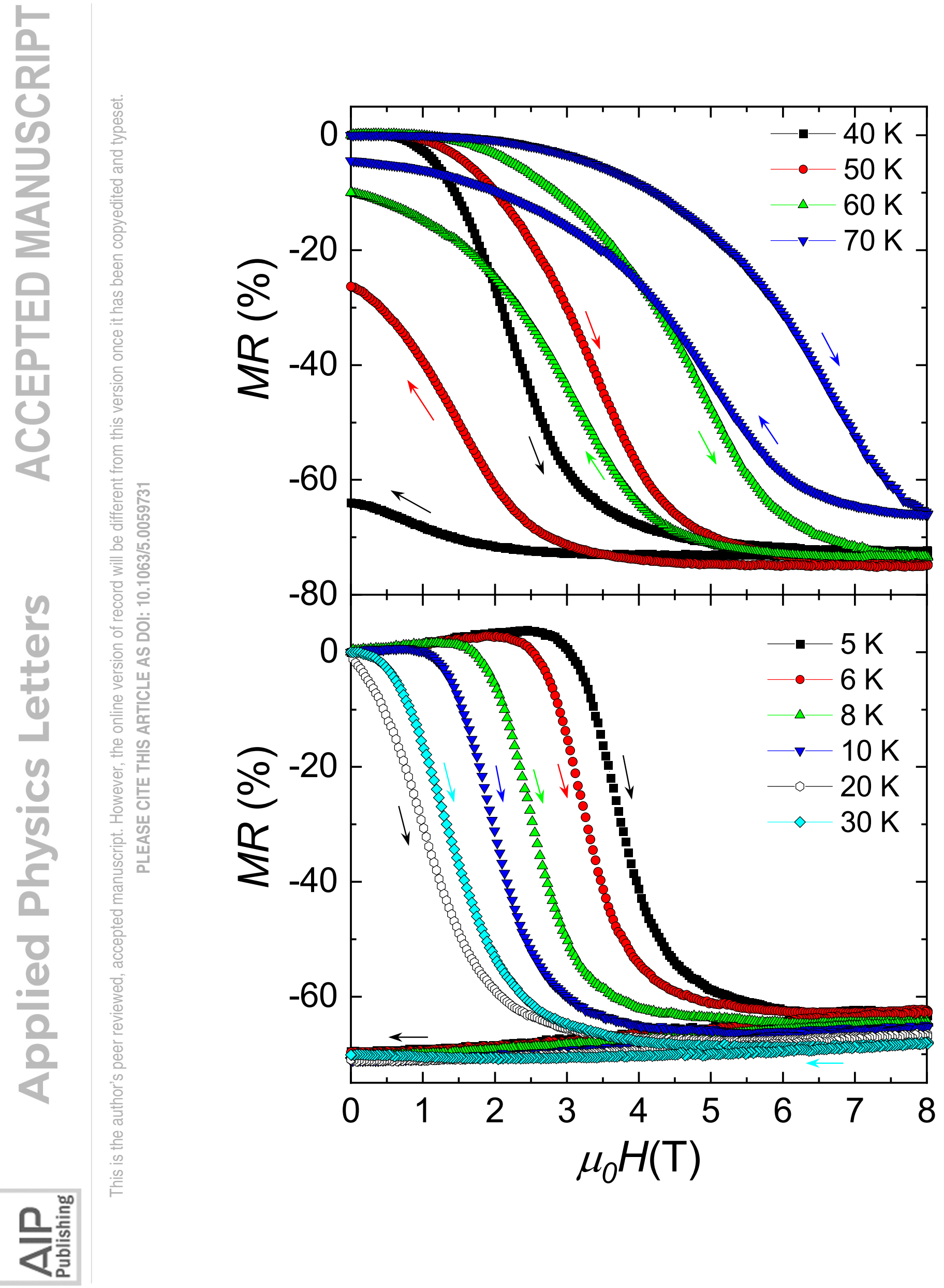


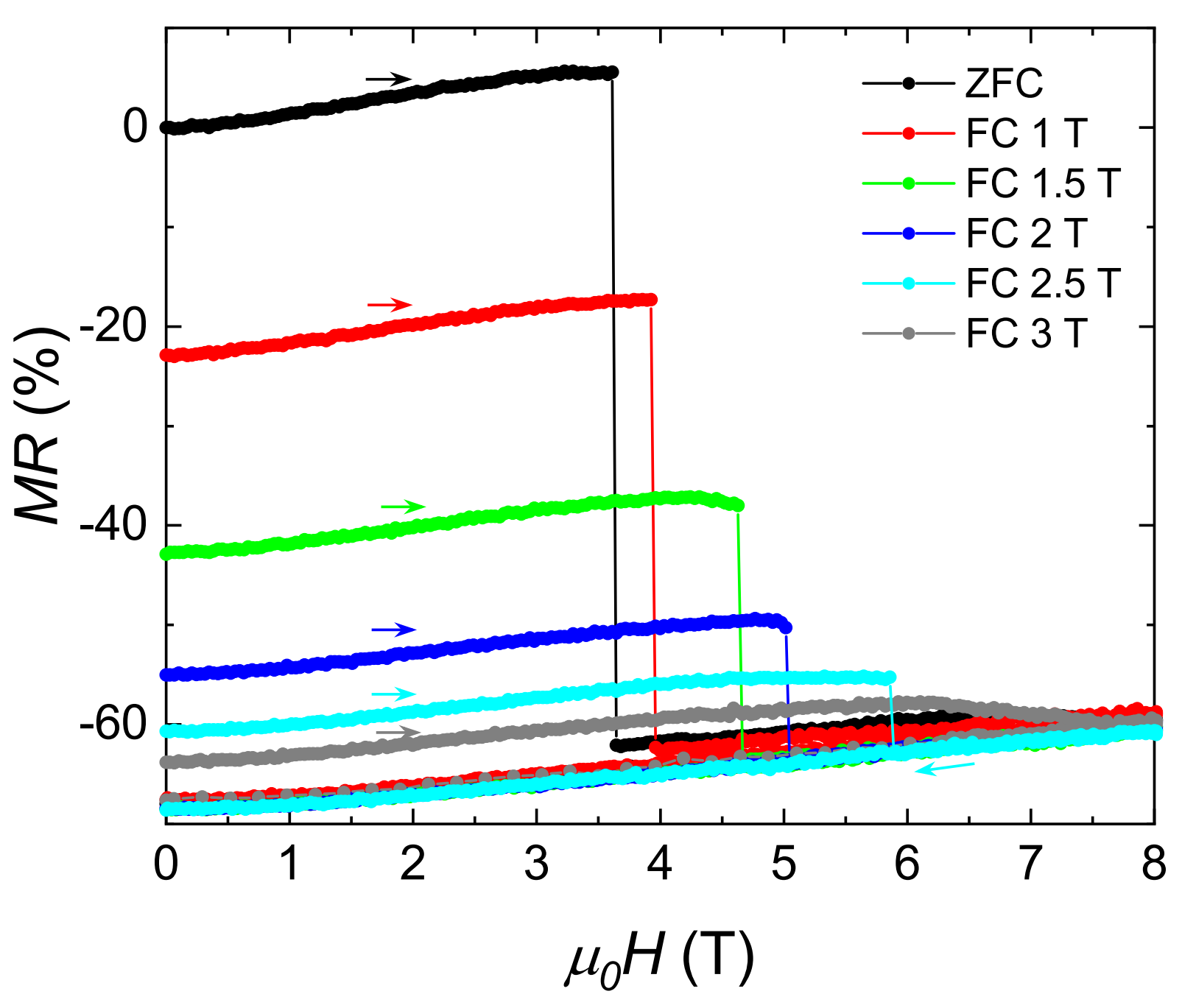

兄旁产 


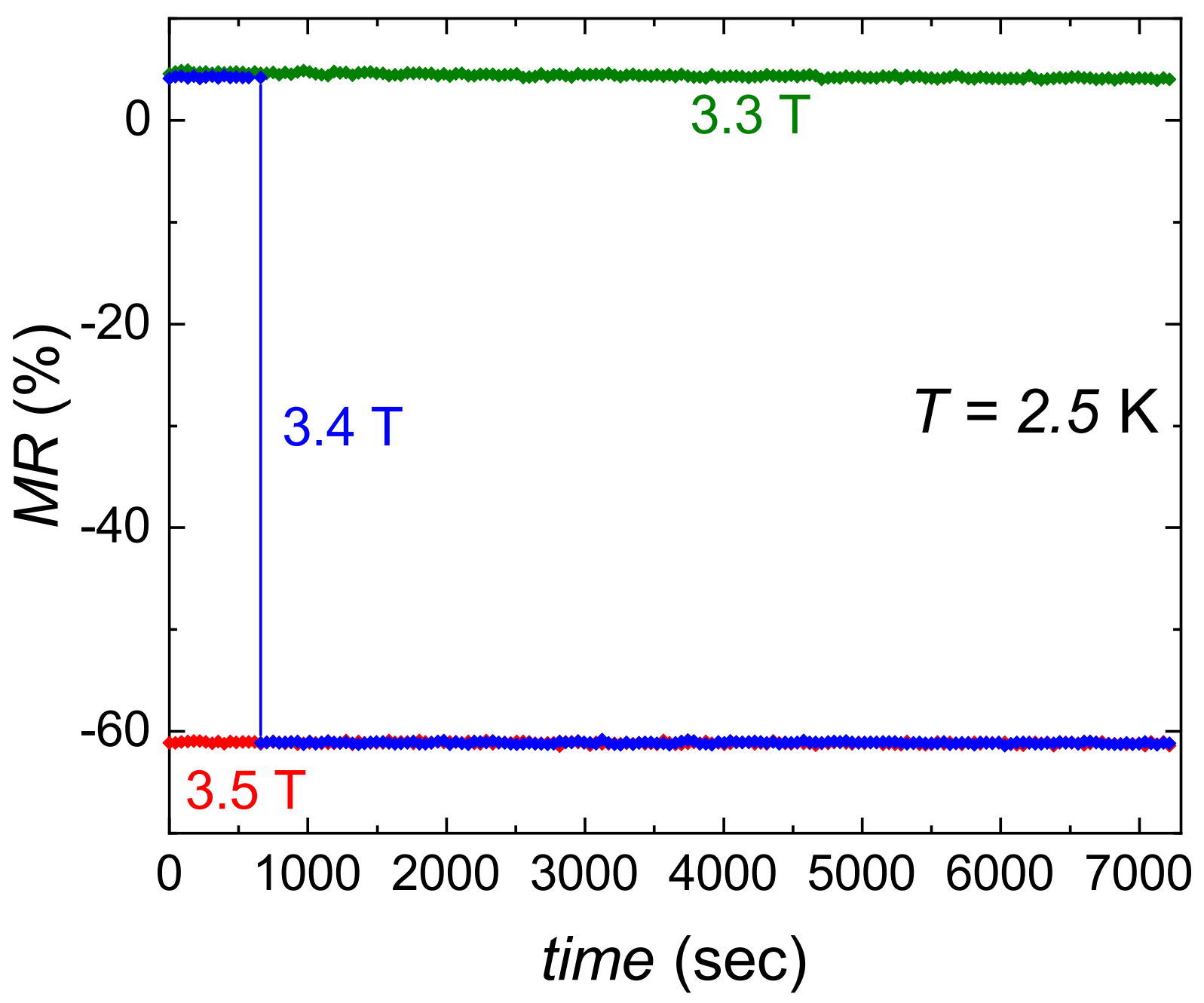

는 